\section{EDUCATION}

Research, Inovortion and Solutions on-line ${ }^{\Theta}$
PSYCHOLOGY

I+D+i
Electronic Journal of Research

in Educational Psychology

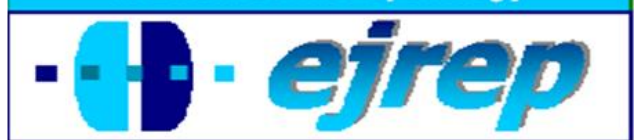

\title{
Relación entre las necesidades psicológicas del estudiante, los enfoques de aprendizaje, las estrategias de evitación y el rendimiento
}

\section{Fernando Doménech Betoret ${ }^{1}$, Amparo Gómez Artiga ${ }^{2}$}

${ }^{1}$ Developmental and Educational Psychology, Universitat Jaume I, Castellón

${ }^{2}$ Developmental and Educational Psychology, Universitat de València (Estudi General), València

\section{España}

Correspondencia: Dr. Fernando Doménech Betoret, Psicología Evolutiva y de la Educación. Universitat Jaume I, 12071 Castellón, España. E-mail: betoret@ psi.uji.es. Tel.: +34 964/729550, Fax: +34 964/729262.

(C) Education \& Psychology I+D+i and Editorial EOS (Spain) 


\section{Resumen}

Introducción. Este estudio examina la relación entre las necesidades psicológicas básicas de los estudiantes (autonomía, competencia, relación y pertenencia), enfoques de aprendizaje (profundo y superficial), estrategias de evitación (evitación al esfuerzo y desafíos, evitación en pedir ayuda, y evitación de la novedad) y rendimiento, en varias situaciones educativas universitarias.

Método. La muestra estaba compuesta por 157 estudiantes universitarios. Para medir los constructos seleccionados se utilizaron escalas de auto-informe. La relación entre dichos constructos se examinó a través de modelos de ecuaciones estructurales.

Resultados. En general, se ha constatado que cuando las necesidades básicas de los estudiantes son satisfechas promueven la utilización de enfoques de aprendizaje profundo, lo que a su vez repercute en una menor utilización de estrategias de evitación y un mayor rendimiento escolar. Por el contrario, cuando no son satisfechas promueven a la utilización de enfoques de aprendizaje superficial, lo que a su vez repercute en una mayor utilización de estrategias de evitación y un menor rendimiento escolar.

Conclusions. Los resultados obtenidos sugieren que los enfoques de aprendizaje y las estrategias de evitación pueden jugar un papel mediador entre las necesidades psicológicas de los estudiantes y el rendimiento escolar. Por último, se discuten las implicaciones prácticas que pueden tener estos hallazgos en la educación universitaria.

Keywords. Necesidades psicológicas, enfoques de aprendizaje, estrategias de evitación, rendimiento escolar. 


\title{
The Relationship among Student Basic Need Satisfaction, Approaches to Learning, Reporting of Avoidance Strategies and Achievement
}

\begin{abstract}
Introduction. This study examines the relationship between student basic need satisfaction (autonomy, competence, relatedness and belonging), their reporting of approaches to learning (deep and surface), their reporting of avoidance strategies (avoidance of effort and challenge, avoidance of help seeking and preference to avoid novelty) and achievement in subject-matter domains.

Method. The sample was composed of 157 Spanish undergraduate students. Self-report questionnaires were used to measure the construct selected for this study and their interrelationships were examined using structural equation procedures.

Results. In general terms, we have proved that basic needs satisfaction, when satisfied, encourage the use of the deep approach to learning and, in turn, the decrease of students' avoidance strategies. In contrast, when these needs are not satisfied, the use of the surface approach to learning is encouraged, consequently, leads to an increase in students' avoidance strategies and achievement.
\end{abstract}

Conclusions. The results suggest that approaches to learning and avoidance strategies may play a mediator role between student psychological needs and student achievement. Implications for pedagogical practice are discussed.

Keywords. Psychological needs, approaches to learning, avoidance strategies, student achievement. 


\section{Introducción}

Este estudio examina la relación entre las necesidades psicológicas básicas de los estudiantes (autonomía, competencia, relación y pertenencia), enfoques de aprendizaje (profundo y superficial), estrategias de evitación (evitación al esfuerzo y desafíos, evitación en pedir ayuda, y evitación de la novedad) y rendimiento en estudiantes universitarios españoles. Se pretende profundizar en el papel que desempeñan las necesidades psicológicas en el aprendizaje del estudiante y de cómo la satisfacción de las mismas puede potenciar el rendimiento académico. La importancia de este estudio es triple: en primer lugar, puede ayudar a tender puentes entre los diferentes ámbitos de la Psicología de la Educación; en segundo lugar, al examinar las conexiones entre las variables consideradas, puede ayudar a comprender el proceso por el cual la satisfacción de las necesidades básicas afecta al rendimiento; y en tercer lugar, por las implicaciones prácticas en la enseñanza y el aprendizaje que de este estudio se pueden derivar. Así pues, además de ampliar investigaciones anteriores realizadas en este campo (Turner, Meyer, Anderman, Midgley, Gheen, \& Kang, 2002; Black \& Deci, 2000), la presente investigación nos puede ayudar a explicar las conexiones entre estrategias de evitación, enfoques de aprendizaje, y rendimiento en situaciones educativas universitarias, así como contribuir a explicar por qué los estudiantes utilizan estrategias negativas en su proceso de aprendizaje. En este sentido, puede proporcionar información útil sobre cómo mejorar el aprendizaje y rendimiento de los estudiantes universitarios.

\section{Satisfacción de las necesidades psicológicas básicas del estudiante}

La Teoría de la Autodeterminación -TAD- (Deci and Ryan 1985, 2000), constituye una teoría organísmica sobre la motivación humana óptima, muy respaldada por los numerosos estudios realizados en el campo de la educación durante las últimas tres décadas, especialmente en Enseñanza Primaria y Secundaria. (Ryan \& Stiller, 1991; Sheldon \& Biddle, 1998). Según la TAD, existen tres necesidades psicológicas básicas (autonomía, competencia y relación) que socavan o apoyan la motivación intrínseca de las personas y les llevan a actuar de una forma determinada. Por ejemplo, los estudios realizados en el campo de la educación en relación con esta teoría han mostrado que el apoyo a la autonomía del estudiante por parte del profesor es un factor importante para maximizar su aprendizaje, su crecimiento personal y su madurez. 
La Autonomía se da cuando la persona siente que es la causante de su conducta (Deci \& Ryan, 1985). “Autonomía no significa independencia o libertad total, sino más bien aceptación interna y compromiso con la conducta motivada. Apoyar la autonomía significa ponerse en el lugar del estudiante, ofrecer opciones, y dar una explicación racional cuando no sea posible dar otra opción" (Filak \& Sheldon, 2003, p. 235). La Competencia se da cuando uno se siente eficaz con su conducta. Es cercana a la auto-eficacia y puede reconocerse cuando una persona emprende nuevas tareas que le suponen un desafío y trata de dominarlas. "Apoyar la competencia significa transmitir a los estudiantes confianza en sus destrezas para superar retos, guiar con sensibilidad y dar feedback (Filak \& Sheldon, 2003, p. 237). La Relación se produce cuando una persona se siente vinculado o comprendido por los demás. Este constructo es similar al de la necesidad de pertenencia propuesto por Baumeister y Leary (1995), pero más general, ya que incluye tanto las relaciones interpersonales como las relaciones de grupo (Filak \& Sheldon, 2003). Apoyar la relación significa proporcionar aceptación, respeto y cariño.

Según la TAD, cuando estas tres necesidades están satisfechas, estimulan el bienestar psicológico y permiten a los alumnos conseguir un rendimiento académico óptimo. Por el contrario, si no lo están, los alumnos no consiguen desarrollarse completamente. Algunas investigaciones anteriores (Reis, Sheldon, Gable, Roscoe, \& Ryan, 2000; Sheldon, Ryan, \& Reis, 1996; Sheldon, Elliot, Kim, \& Kasser, 2001) han constatado de forma empírica estos supuestos. Otras investigaciones previas también han demostrado cómo la satisfacción de las necesidades psicológicas básicas producen un efecto positivo sobre el rendimiento (Black \& Deci, 2000); sin embargo, son escasos los trabajos centrados en el estudio de la relación entre las necesidades psicológicas básicas del estudiante y su rendimiento en situaciones educativas específicas. Por último, la TAD sugiere además que la satisfacción de las tres necesidades psicológicas básicas mejora la motivación intrínseca de los empleados, lo que, a su vez, repercute en resultados importantes, como un rendimiento eficaz, especialmente en las tareas que requieran creatividad, flexibilidad cognitiva y comprensión de conceptos" (Gagné \& Deci, 2005, p. 337).

\section{Enfoques de Aprendizaje}

Existe un consenso general entre los autores en adoptar la clasificación de enfoque superficial y profundo del aprendizaje (Biggs, 1987, Ramsden \& Entwistle, 1981). El enfoque profundo se caracteriza por una preocupación de buscar el sentido y comprensión de la 
materia que se estudia, mediante la elaboración y transformación de la misma (Dart, Burnett, Purdie, Boulton-Lewis, Campbell, \& Smith, 2000). Este enfoque de aprendizaje se asocia a la enseñanza constructivista (Biggs \& More, 1993). Con el enfoque superficial, la materia objeto de estudio se reproduce utilizando procedimientos rutinarios y memorísticos, y se relaciona con el modelo de enseñanza tradicional transmisiva en el que los estudiantes adoptan roles pasivos (Dart, et al., 2000). Algunos autores han verificado también la existencia de cierta relación entre el tipo de motivación y el enfoque de aprendizaje. Así, el tipo de motivación predominante del estudiante podría estar relacionado de una manera significativa con el uso de ciertas estrategias de aprendizaje y por consiguiente con el enfoque que el estudiante utiliza. (Biggs, 1989, Entwistle, 1987, Schmeck 1988). La relación motivo-estrategia que compone y define cada enfoque explica el método de aprendizaje seguido por los estudiantes que adoptan uno u otro enfoque. De este modo, los estudiantes que adoptan un enfoque superficial desarrollan ciertas estrategias diseñadas a aprender la información de forma mecánica y repetitiva para reproducirla después a su debido tiempo. En cambio, los estudiantes que adoptan un enfoque profundo muestran un interés intrínseco implicándose en la materia que están aprendiendo. Estos estudiantes se caracterizan por su interés en alcanzar una comprensión significativa de los contenidos de la materia, y para ello adoptan estrategias que les lleven a comprender el significado inherente de la misma, e intentan relacionarla con sus conocimientos previos. Finalmente, se constató que los enfoques de aprendizaje predicen el rendimiento académico (Diseth \&Martinsen, 2003).

\section{Conductas de evitación}

Según Covington (1992), cuando los estudiantes quieren proteger su autoestima porque no están seguros de tener las habilidades necesarias para ser competentes en la escuela, pueden desarrollar estrategias que desvíen la atención de su habilidad. De esta manera protegen su imagen pública de competencia. Existen diversas estrategias al alcance de los estudiantes para desviar la atención de sus habilidades, tales como evitar pedir ayuda, evitar tareas escolares nuevas, evitar esfuerzos y desafíos, reducir el esfuerzo (autoimpedimento), etc. Aunque estas estrategias puedan proteger al estudiante de los juicios negativos de sus profesores y compañeros, pueden también afectar de manera negativa a su trabajo. Un estudio anterior (Nichols \& Miller, 1984) ha revelado que los comportamientos de evitación son más frecuentes en adolescentes jóvenes porque a esta edad cambian el concepto que tienen de habilidad; pasan de ver la habilidad como algo que se puede modificar con esfuerzo, a algo que es permanente. Si los estudiantes perciben que su profesor trata de dar 
respuesta a sus necesidades psicológicas básicas, se sentirán más satisfechos y más cómodos en la clase, por consiguiente no se preocuparán tanto de proteger su autoestima.

Necesidades psicológicas básicas, enfoques de aprendizaje y comportamientos de evitación

Hasta la fecha, que nosotros sepamos, no existen estudios que hayan examinado la relación entre la satisfacción de las necesidades del estudiante con los enfoques de aprendizaje y los comportamientos de evitación, pero se pueden establecer conexiones entre ellos. Los estudios han revelado que la percepción que tienen los alumnos de sus entornos de aprendizaje tienen una influencia significativa en su forma de enfocar el aprendizaje e incluso en la calidad de sus resultados (e.g. Doyle, 1977; Fraser, 1989, 1998; Ramsden, 1992; Waxman, 1991). Más concretamente, en las últimas dos décadas se han realizado numerosos estudios que relacionan los enfoques de aprendizaje con la percepción del entorno psicológico en el aula (e.g., Dart, Burnett, Boulton-Lewis, Campbell, Smith, \& McCrindle, 1999; CleaveHogg \& Rothman, 1991; Ramsden, Martin, \& Bowden, 1989; Yuen-Yee \& Watkins, 1994). Según la TAD, el entorno en el aula (estrategias de enseñanza incluidas) debe diseñarse con el fin de satisfacer las necesidades psicológicas de los estudiantes, ya que así se mejorará la motivación intrínseca de su aprendizaje. Como Valas y Sovik (1993) postulan: "to what extent a student is intrinsically motivated for an activity depends on how far this activity and the related context can meet these needs" [el grado que manifiesta un estudiante estar motivado intrínsecamente por una actividad, depende de hasta que punto esa actividad y el contexto relacionado pueden satisfacer sus necesidades] (p. 282). Por consiguiente, se puede inferir que la percepción de los estudiantes sobre la satisfacción de sus necesidades psicológicas (entorno de aprendizaje) puede relacionarse con el enfoque de aprendizaje que adopte. Asimismo, la TAD postula que la satisfacción de las necesidades psicológicas básicas proporciona los nutrientes para la motivación intrínseca y la internalización (Gagné \& Deci, 2005) y, como exponíamos anteriormente, el tipo de motivación predominante en el estudiante podría estar relacionado de manera significativa con la utilización de de ciertas estrategias de aprendizaje y, en consecuencia, con enfoque de aprendizaje que utilice (Biggs, 1989, Entwistle, 1987, Schmeck 1988). Por otra parte, la relación estrategia-motivo, que constituye y define cada enfoque, sugiere que las conexiones entre necesidades psicológicas y enfoques de aprendizaje pueden ser importantes. Si las condiciones que conducen a la satisfacción de las necesidades psicológicas básicas facilitan la motivación intrínseca (Deci \& 
Ryan 2000), y la motivación intrínseca implica un enfoque profundo (ya que el estudiante que adopta un enfoque profundo muestra un interés intrínseco y se involucra en la materia de estudio), se pueden inferir conexiones positivas entre las necesidades psicológicas y el enfoque profundo del aprendizaje. Estudios anteriores confirman también que la motivación intrínseca está asociada a un mejor aprendizaje, a un mayor rendimiento y al bienestar psicológico (Valas \& Sovik, 1993). Por el contrario, se pueden inferir conexiones negativas con el enfoque superficial, ya que el estudiante que adopta un enfoque superficial desarrolla ciertas estrategias orientadas a aprender la información de forma mecánica y repetitiva con el fin de reproducirla en el momento adecuado.

Como hemos señalado anteriormente, la satisfacción de las necesidades psicológicas potencia la motivación intrínseca de los estudiantes y la motivación intrínseca "se manifiesta como curiosidad e interés, lo que motiva la implicación en la tarea incluso cuando no hay un refuerzo o apoyo exterior...” (Ryan, Connell, \& Grolnick, 1992, p.170). Por lo tanto, se puede suponer que los estudiantes con motivación intrínseca estarán más implicados en su proceso de aprendizaje y por consiguiente, las estrategias de evitación se verán reducidas. Por lo contrario, cuando no se satisfacen las necesidades psicológicas, la motivación intrínseca puede convertirse en desmotivación (el polo opuesto en el continuo de la autodeterminación). Por otro lado, según la TAD, las personas tienden a estar desmotivadas cuando carecen de un sentido de la eficacia o de un sentido de control con respecto a unos resultados deseados (Deci \& Ryan 2000). Por lo tanto, basándonos en la TAD, la utilización de las estrategias de evitación podría explicarse como una consecuencia de la desmotivación de los estudiantes. Desde esta lógica, pueden inferirse conexiones negativas entre las necesidades psicológicas y las estrategias de evitación.

\section{Objetivos e hipótesis}

De acuerdo con los planteamientos expuestos y el modelo conceptual propuesto, el objetivo de este estudio es examinar la relación entre la satisfacción de las necesidades psicológicas básicas de los estudiantes, las estrategias de evitación y el rendimiento en varias asignaturas universitarias. La relación entre las variables hipotetizadas está estructurada en tres fases secuenciales en las que la satisfacción de las necesidades psicológicas básicas (dependiendo de la percepción que tenga el alumno de su entorno de aprendizaje) se considera variable de entrada o input, los enfoques de aprendizaje de los estudiantes y las estrategias de evitación se consideran variables del proceso, y el rendimiento escolar se toma como variable 
de salida o output. Hemos considerado las necesidades psicológicas como factor de orden superior (variable latente) en lugar de tomarlas como predictores independientes, dadas las importantes correlaciones que se observaron entre las mismas. El modelo general hipotetizado se muestra en la Figura 1.
Necesidades del estudiante

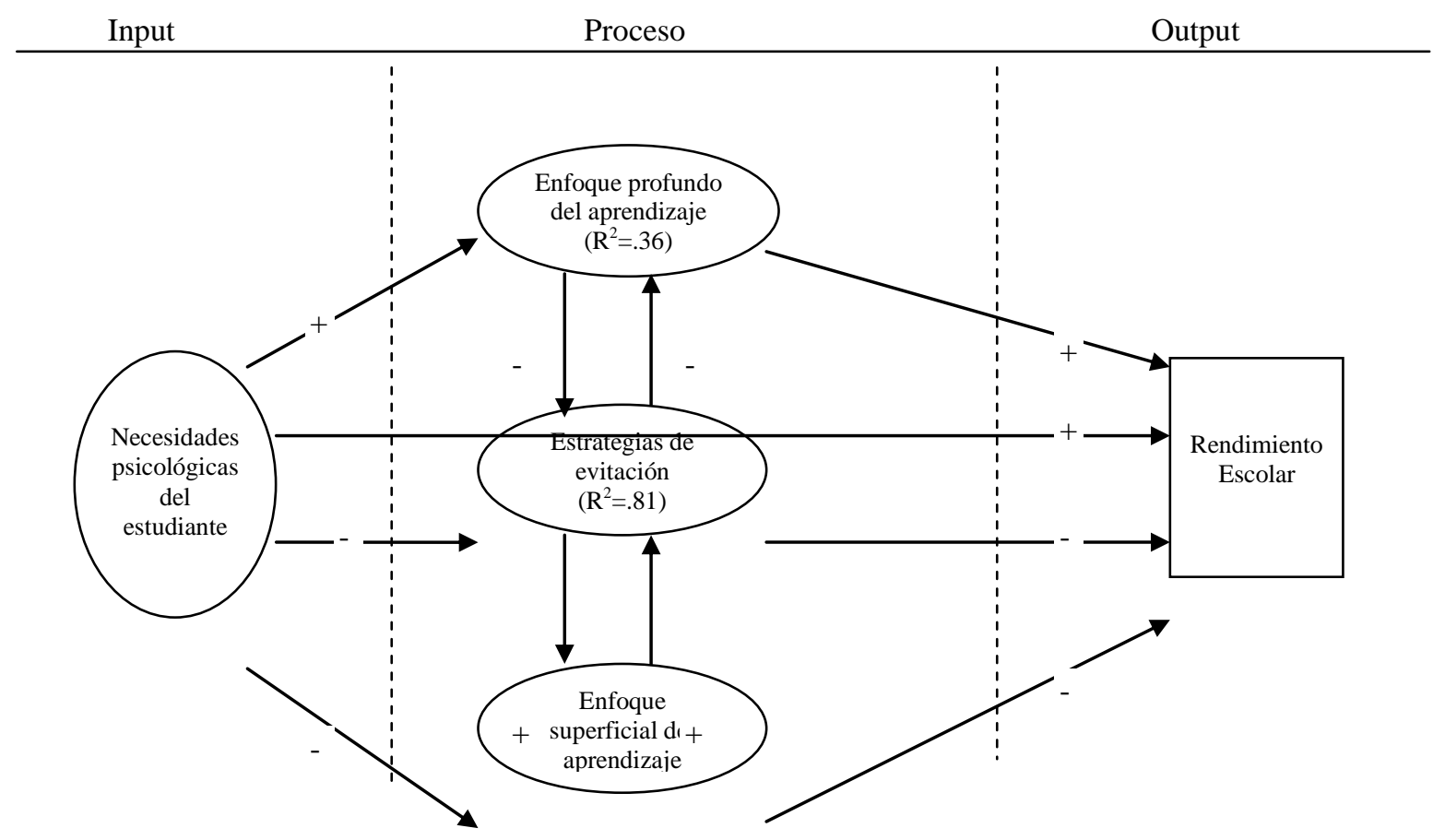

Figura 1. Estructura del modelo general hipotetizado

Basándonos en la configuración del modelo propuesto, predecimos (Hipótesis 1) que la satisfacción de las necesidades psicológicas del alumno tendrá una relación positiva con el enfoque profundo y negativa con el enfoque superficial. A su vez, tanto el enfoque profundo como el superficial estarán relacionados con el rendimiento, de manera positiva y negativa, respectivamente. Por último, la satisfacción de las necesidades estará relacionada con el rendimiento académico. Pensamos, que si el estudiante percibe que el profesor proporciona apoyo a sus necesidades psicológicas, su motivación intrínseca mejorará, y por consiguiente, el estudiante tenderá a adoptar un enfoque profundo del aprendizaje. Sin embargo, si el estudiante percibe que el profesor no presta apoyo a sus necesidades, tenderá a adoptar un enfoque superficial, y por consiguiente el rendimiento escolar se verá afectado. 
También predecimos (Hipótesis 2) que la satisfacción de las necesidades psicológicas del alumno se relacionarán de manera negativa con las estrategias de evitación (evitación al esfuerzo y desafíos, evitación en pedir ayuda, y evitación de la novedad); a su vez, las estrategias de evitación se relacionarán de forma negativa con el rendimiento escolar. Finalmente, la satisfacción de las necesidades psicológicas básicas se relacionará de manera positiva con el rendimiento académico. Pensamos que cuando los estudiantes perciban que sus necesidades básicas son satisfechas, mayor será su motivación intrínseca. La motivación intrínseca "se manifiesta con la curiosidad y el interés, que estimulan la implicación en la tarea, incluso cuando no haya un refuerzo o apoyo externo..." (Ryan, Connell, \& Grolnick, 1992, p.170). Por lo que, parece lógico, pensar que dichos estudiantes estarán más implicados en su proceso de aprendizaje lo que, a su vez, conducirá a una reducción de las estrategias de evitación, y viceversa. En consecuencia, el rendimiento escolar se verá afectado de forma positiva.

Dado que “ los estudiantes que adoptan un enfoque superficial del aprendizaje: (a) ven la tarea como un trabajo que hay que hacer, una imposición necesaria si se quiere alcanzar otro objetivo - una nota por ejemplo-; (b) ven algunos aspectos o partes de la tarea como discretos y que no guardan relación entre ellos o con otras tareas; (c) se preocupan por el tiempo que les va a llevar hacer la tarea; (d) evitan la implicación personal o de otra índole que la tarea pueda tener; y (e) confían en la memorización, intentando reproducir los aspectos superficiales de la tarea" (Biggs 1987, p. 15), parece lógico pensar que la estrategia de evitación tendrá una relación positiva con el enfoque superficial y una relación negativa con el enfoque profundo. Sin embargo, ya que la presente investigación es un estudio de corte transversal, no podemos suponer causalidad estricta en una dirección específica; es decir, los estudiantes que utilizan estrategias de evitación y siguen un enfoque de aprendizaje de mínimo esfuerzo, probablemente adoptarán un enfoque superficial de aprendizaje, y viceversa. Basándonos en estas consideraciones, se han ampliado los modelos anteriores. Por un lado, la satisfacción de las necesidades psicológicas básicas del estudiante tendrá un efecto positivo sobre el enfoque profundo, y un efecto negativo sobre el enfoque superficial y sobre los enfoques de evitación. También se prevé que el enfoque profundo de aprendizaje tendrá un efecto negativo sobre las estrategias de evitación, mientras que el efecto del enfoque superficial sobre las estrategias de evitación se prevé que sea positivo. Por último, el enfoque profundo tendrá un efecto positivo sobre el rendimiento de los estudiantes; y el enfoque superficial y las estrategias de evitación tendrán un efecto negativo sobre el rendimiento 
(Hipótesis 3). Por otro lado, también se prevé que las estrategias de evitación tengan un efecto positivo sobre el enfoque superficial y un efecto negativo sobre el enfoque profundo. Para finalizar, el rendimiento escolar de los estudiantes se verá afectado de manera positiva por el enfoque profundo y de manera negativa por el enfoque superficial y las estrategias de evitación (Hipótesis 4).

\section{Método}

Participantes y agrupamiento de los estudiantes

La muestra estaba compuesta por 157 estudiantes universitarios, 132 mujeres (84\%) y 25 hombres (15.9\%), pertenecientes a diferentes asignaturas: Psicología de la Instrucción $(\mathrm{n}=84,53.5 \%)$, Bases Psicológicas de la Educación Especial $(\mathrm{n}=50,31.8 \%)$, y Programas y Técnicas de Intervención temprana $(n=23,14.6 \%)$.

Psicología de la Instrucción (PI) es una asignatura anual de 9 créditos (6 teoría, 1.5 práctica y 1.5 laboratorio) obligatoria en la carrera de Psicología. Esta asignatura se imparte en el tercer curso de carrera en la Universidad Jaime I de Castellón (España). Bases Psicológicas de la Educación Especial (BSEE), es una asignatura cuatrimestral obligatoria que se imparte en la carrera de Magisterio en la Universidad Jaime I (España), consta de 4,5 créditos (3 teoría y 1.5 práctica). Programas y Técnicas de Intervención Temprana (PTIT) es una asignatura cuatrimestral obligatoria que se imparte durante el segundo curso de carrera de Psicología de la Educación en la Universitat de València (España), consta de 5.5 créditos (4.5 teoría y 1 práctica).

\section{Medidas}

Las escalas que se enumeran a continuación se utilizaron para recopilar la información de los estudiantes participantes en relación con las variables consideradas en este estudio. En la Tabla 1 se puede ver un resumen del análisis factorial realizado en cada escala.

Escala de Necesidades Psicológicas Básicas (17 ítems). Este instrumento se construyó partiendo de la Escala de Necesidades Psicológicas Básicas desarrollada por Ilardi, Leone, Kasser, y Ryan (1993). Sin embargo, hemos ampliado la estructura original de la escala, que se componía de tres elementos (Autonomía, Competencia y Relación), añadiéndole una cuarta necesidad, la necesidad de Pertenencia, que tiene un notable interés de acuerdo con las investigaciones previas (Goodenow, 1992). La necesidad de Pertenencia hace 
referencia al sentimiento del alumno de pertenecer a un grupo/clase formada por otros alumnos de su mismo curso académico.

Un análisis factorial preliminar (componentes principales con rotación Oblimin) se llevó a cabo para aclarar la interpretación del constructo, ya que presentaban saturaciones importantes (.30 o mayor) en más de un factor. Un segundo análisis factorial (componentes principales con rotación Oblimin) se llevó a cabo con los 17 ítems restantes. Se extrajeron cuatro factores que correspondían a las cuatro necesidades básicas consideradas: Autonomía (4 ítems, $\alpha=0.76$ ), Competencia (4 ítems, $\alpha=0.65$ ), Relación (4 ítems, $\alpha=0.82$ ) y Pertenencia (5 ítems, $\alpha=0.86$ ). Véase el Apéndice para consultar el cuestionario completo. Se utilizaron escalas Likert de cuatro puntos para cada enunciado que iban de 1 (Estoy totalmente en desacuerdo) a 4 (Estoy totalmente de acuerdo)

Escala de enfoques de aprendizaje (20 ítems). Esta escala se basó en el cuestionario bifactorial revisado sobre procesos de estudio, (R-SPQ-2F), desarrollado por Biggs, Kember y Leung (2001). Siguiendo una adaptación para estudiantes universitarios españoles, esta escala se utilizó para evaluar los enfoques de aprendizaje (superficial y profundo) que adoptaban los estudiantes de psicología de la muestra. El cuestionario R-SPQ-2F consta de cuatro subescalas de cinco ítems cada una: motivación profunda (MP), estrategia profunda (EP), motivación superficial (MS) y estrategia superficial (ES). En la escala original, los ítems van de 1 (nunca o casi nunca ciertos para mí) a 5 (siempre o casi siempre ciertos para mí). En la versión española, los ítems van de 1 (Estoy completamente en desacuerdo) a 4 (Estoy completamente de acuerdo). Para comprobar la adecuación a esta muestra del cuestionario de estructura factorial R-SPQ-2F (previamente traducido al español y adaptado para esta investigación), se empleó el procedimiento descrito por Burnett y Dart (1997). Esto dio como resultado que se mantuvieran tres ítems para la subescala de la MP, cuatro ítems para la subescala EP, cuatro para la subescala MS, y tres para la subescala ES (véase Justicia, Pichardo, Cano, Berbén \& De la Fuente, 2008, para más detalles sobre la estructura validada del R-SPQ-2F con ejemplos españoles). La consistencia interna de las cuatro subescalas era en general aceptable ( $\alpha=0.68, \alpha=0.65, \alpha=0.83$, y $\alpha=0.61$, respectivamente) y parecida a la escala original, a excepción de la escala MS que era más elevada en este caso). Los ítems utilizados en cada escala pueden verse en el Apéndice.

Escala de estrategias de evitación (15 ítems). Esta escala consta de tres subescalas: evitación de la novedad (5 ítems), evitación en pedir ayuda (5 ítems) evitación al esfuerzo y 
desafíos (5 ítems). Las dos primeras escalas de estrategias de evitación (evitación de la novedad y evitación en pedir ayuda) empleadas en este estudio son una adaptación de las utilizadas por Turner, Meyer, Anderman, Midgley, Gheen, y Kang (2002) con alumnos de sexto de primaria, mientras que la tercera, evitación al esfuerzo y desafíos, fue diseñada expresamente para este estudio. Los participantes respondieron a los ítems en una escala que iba de 1 (Totalmente falso) a 5 (Muy verdadero). Se llevó a cabo un análisis factorial de los componentes principales con rotación oblimin con los catorce ítems conjuntamente. Este análisis arrojó tres factores para esta muestra de acuerdo con las tres evitaciones consideradas: Evitación de la Novedad, (formada por 5 ítems, $\alpha=0.76$ ), Evitación búsqueda de ayuda (formada por 5 ítems, $\alpha=.73$ ) y Evitación Esfuerzo y Desafío (formada por 5 ítems, $\alpha=.84)$. Véase en el Apéndice el cuestionario completo.

Medida del rendimiento. El rendimiento académico de los estudiantes se midió con las puntuaciones obtenidas por los alumnos en una prueba individual realizada para evaluar su conocimiento teórico de la asignatura impartida. Las puntuaciones iban de 1 (mínimo) a 10 (máximo)

\section{Procedimiento}

Los cuestionarios se administraron durante dos sesiones de clase, al final del Segundo cuatrimestre, y fueron recogidos por los autores. Tras proporcionar información verbal sobre el trabajo de investigación y aclarar todas las dudas, se pidió a los participantes que respondieran a todos los ítems de las escalas. Todos los participantes lo hicieron de forma voluntaria.

\section{Análisis estadístico}

Para llevar a cabo el análisis estadístico se utilizaron los programas SPSS (Norusis, 1999) y EQS (Bentler, 1990, 1995). El primer programa (SPSS) se utilizó para evaluar la validez del constructo y la consistencia interna de las escalas, así como el análisis de correlaciones bivariadas de Pearson entre las variables consideradas. El segundo programa (EQS) nos permitió examinar las relaciones estructurales hipotetizadas (método de máxima verosimilitud), de acuerdo con el modelo teórico propuesto. 


\section{Resultados}

Estructura y consistencia interna de las escalas

La media, la desviación típica, la fiabilidad y la estructura de la escala se muestran en la Tabla 1. Se llevó a cabo un análisis factorial de componentes principales con rotación oblimin en todos los conjuntos de ítems de cada escala utilizada para evaluar su validez de constructo. En general, el análisis confirmó la estructura original y la configuración de las escalas y subscalas utilizadas, y el test de Cronbach $\alpha$ mostró una fiabilidad alfa aceptable ( .61 a .86). Como se puede ver en la Tabla 1, los valores más bajos de alfa se obtuvieron con las subescalas de los enfoque de aprendizaje (entre .61 y .79). Sin embargo, fueron parecidos a los de la escala original (véase la escala de validación en Biggs, Kember \& Leung, 2001) y a estudios posteriores (véase Leung \& Kember, 2003). Se ha de tener en cuenta también que el número de ítems en la escala afecta los valores de alfa (Lord \& Novick, 1968), y que las subescalas a las que nos estamos refiriendo están formadas por tres o cuatro ítems. Además debe señalarse que la fiabilidad se puede subestimar cuando se mide con una escala multidimensional (Schmitt, 1996). Se obtuvo una medida del constructo al promediar los ítems incluidos en cada factor.

Tabla 1. Resumen del análisis factorial y de la consistencia interna de las escalas $(\mathbf{n}=157)$.

\begin{tabular}{|c|c|c|c|c|c|c|c|}
\hline \multirow{2}{*}{\multicolumn{2}{|c|}{$\frac{\mathrm{F}}{\text { Necesidades de los estudiantes }}$}} & \multirow{2}{*}{ Factores } & \multirow[t]{2}{*}{ Ítems } & \multirow[t]{2}{*}{ Media } & \multicolumn{2}{|c|}{ S. D. \%Varianza } & Cronbach's $\alpha$ \\
\hline & & & & & & & \\
\hline Pertenencia & & 1 & 5 & 2.92 & 0.62 & $28,82 \%$ & 0.86 \\
\hline Autonomía & & 1 & 4 & 3.31 & 0.53 & $14.57 \%$ & 0.76 \\
\hline Relación & & 1 & 4 & 3.39 & 0.57 & $11,24 \%$ & 0.82 \\
\hline Competencia & & 1 & 4 & 2.95 & 0.56 & $6,83 \%$ & 0.65 \\
\hline Total & & 4 & 17 & & & $61,47 \%$ & \\
\hline \multicolumn{8}{|l|}{ Enfoques de aprendizaje } \\
\hline Motivación superficial & 1 & 4 & 1.95 & 0.67 & $37,15 \%$ & 0.79 & \\
\hline Estrategia Superficial & 1 & 3 & 2.05 & 0.68 & $9,90 \%$ & 0.61 & \\
\hline Motivación Profunda & & 1 & 3 & 2.97 & 0.66 & $9,43 \%$ & 0.68 \\
\hline Estrategia Profunda & & 1 & 4 & 2.29 & 0.65 & $7,38 \%$ & 0.66 \\
\hline Total & & 4 & 14 & & & $63.88 \%$ & \\
\hline \multicolumn{8}{|l|}{ Estrategias de Evitación } \\
\hline Esfuerzo/rendimiento & 1 & 5 & 2.26 & 0.76 & $31,81 \%$ & 0.84 & \\
\hline Evitación novedad & 1 & 5 & 2.33 & 0.75 & $15,34 \%$ & 0.76 & \\
\hline Evitación búsqueda ayu & & 1 & 5 & 2.23 & 0.68 & $11,01 \%$ & 0.73 \\
\hline Total & & 3 & 15 & & & $58.16 \%$ & \\
\hline
\end{tabular}




\section{Correlación entre variables}

Posteriormente, se calculó también la correlación bivariada de Pearson entre los constructos. En general, las correlaciones obtenidas (véase la Tabla 2) se ajustaban a nuestras expectativas. Por un lado, se podían ver correlaciones significativas y positivas entre la mayoría de la satisfacción de las necesidades básicas de los estudiantes (competencia, relación y pertenencia) y el enfoque profundo, mientras que se observaron correlaciones significativas y negativas entre la mayoría de la satisfacción de las necesidades básicas de los estudiantes (competencia, relación y pertenencia) y el enfoque superficial. Sin embargo, la autonomía no se asociaba a los enfoques de aprendizaje. Por otro lado, las correlaciones entre las necesidades básicas de los estudiantes y las estrategias de evitación (evitación al esfuerzo y desafíos, evitación en pedir ayuda, y evitación de la novedad) iban en la misma dirección, y como en el caso anterior, la necesidad de autonomía no se veía asociada con las estrategias de evitación. Para finalizar, nos gustaría señalar que se obtuvieron correlaciones bajas entre la satisfacción de las necesidades básicas de los estudiantes y el rendimiento académico, en cambio se observaron correlaciones significativas entre los enfoques de aprendizaje (sobre todo con las estrategias superficiales; $\mathrm{r}=-.334, \mathrm{p}<.001)$ y rendimiento, y también entre algunas estrategias de evitación (sobre todo con evitación de esfuerzo y desafío; $r=-.380, p<$. 001) y rendimiento. Véase la Tabla 2 para más detalles. 
Tabla 2. Correlación bivariada de Pearson entre los constructos considerados ( $\mathrm{n}=157)$.

\begin{tabular}{|c|c|c|c|c|c|c|c|c|c|c|c|c|}
\hline & 1.AUTON & 2.COMP & 3.RELAC & 4.PERTEN & 5. MP & 6. MS & 7.SM & 8.ES & 9.NOVE & 10.AYUDA & 11.ESFZO & 12.RENDIM \\
\hline 1. NECESIDAD AUTONOMÍA & 1 & & & & & & & & & & & \\
\hline 2. NECESIDAD COMPETENCIA & $.175(*)$ & 1 & & & & & & & & & & \\
\hline 3. NECESIDAD RELACIÓN & $.214(* *)$ & $.430(* *)$ & 1 & & & & & & & & & \\
\hline 4. NECESIDAD_PERTENENCIA & .057 & $.355(* *)$ & $.343(* *)$ & 1 & & & & & & & & \\
\hline 5. ENFOQUE_MP & .081 & $.259(* *)$ & $.233(* *)$ & $.256(* *)$ & 1 & & & & & & & \\
\hline 6. ENFOQUE_MS & -.033 & $.208(* *)$ & .135 & $.158(*)$ & $.256(* *)$ & 1 & & & & & & \\
\hline 7. ENFOQUE_SM & .040 & $-.549(* *)$ & $-.347(* *)$ & $-.282(* *)$ & $-.289(* *)$ & $-.254(* *)$ & 1 & & & & & \\
\hline 8. ENFOQUE_ES & -.030 & $-.381(* *)$ & $-.261(* *)$ & $-.169(*)$ & $-.379(* *)$ & $-.274(* *)$ & $.691(* *)$ & 1 & & & & \\
\hline 9. EVITACIÓN NOVEDAD & -.041 & $-.289(* *)$ & -.098 & $-.188(*)$ & -.063 & -.041 & $.448(* *)$ & $.292(* *)$ & 1 & & & \\
\hline 10. EVITACIÓN BUSCAR AYUDA & .051 & $-.199(*)$ & $-.249(* *)$ & -.119 & -.067 & -.115 & $.395(* *)$ & $.290(* *)$ & $.201(*)$ & 1 & & \\
\hline 11. EVITACIÓN ESFZO-DESAFIO & .151 & $-.482(* *)$ & $-.299(* *)$ & $-.232(* *)$ & -.120 & $-.383(* *)$ & $.737(* *)$ & $.590(* *)$ & $.328(* *)$ & $.345(* *)$ & 1 & \\
\hline 12. RENDIMIENTO & .066 & $.243(* *)$ & $.161\left(^{*}\right)$ & .147 & .103 & $.281(* *)$ & $-.258(* *)$ & $-.334(* *)$ & -.128 & $-.294(* *)$ & $-.380(* *)$ & 1 \\
\hline
\end{tabular}

$* * \mathrm{p}<.01 . \quad * \mathrm{p}<.05$ 
Puesto que la muestra se tomó de diferentes clases (Psicología de la Instrucción, Bases Psicológicas de la Educación Especial, y Programas y Técnicas de Intervención Temprana) pertenecientes a dos universidades distintas, comprobamos si había diferencias significativas entre los grupos con respecto a las estrategias de evitación, enfoques de aprendizaje y necesidades. Para estudiar si existían diferencias significativas entre los tres grupos de universitarios con respecto a las estrategias de evitación, enfoques de aprendizaje y necesidades psicológicas, se llevaron a cabo análisis de la varianza (ANOVA). Posteriores comparaciones post hoc mediante el método Bonferroni mostraron algunas diferencias significativas en dos estrategias de evitación (evitación al esfuerzo y desafíos, y evitación de la novedad) entre los grupos de PTIT y BPEE $(\mathrm{p}=.02, \mathrm{p}=.003$, respectivamente; lo que indica que los estudiantes de PTIT mostraron una utilización más significativa de estas estrategias), $\mathrm{y}$ en las tres estrategias de evitación entre PI y BPEE $(\mathrm{p}=.026, \mathrm{p}=.020, \mathrm{p}=.000$, respectivamente; lo que indica que los estudiantes de PI mostraron una utilización más significativa de estas estrategias). También se obtuvieron diferencias significativas en el enfoque profundo (MP y EP) entre los grupos PI y BPEE ( $p=0.000, p=0.000$, respectivamente; lo que indica que los estudiantes del grupo BPEE mostró un uso más significativo de las estrategias profundas), y entre los grupos PI y PTIT ( $\mathrm{p}=.000, \mathrm{p}=.000$ respectivamente; lo que indica que los estudiantes del grupo BPEE mostró un uso más significativo de las estrategias profundas). Sin embargo, no se hallaron diferencias significativas en necesidades psicológicas entre los tres grupos, a excepción de Relación entre los grupos PI y PTIT ( $\mathrm{p}=.011$; lo que indica que los estudiantes de PTIT tenían una percepción significativamente más positiva de satisfacer esta necesidad).

\section{Modelos de ecuaciones estructurales}

La evaluación de los Modelos hipotetizados se comprobaron con el programa EQS (Bentler, 1995). Este proceso se llevó a cabo en dos etapas. En primer lugar, se calcularon los índices de ajuste global para comprobar el grado en que cada modelo reproduce las relaciones que existen en la matriz de correlación de los datos empíricos. En segundo lugar se analizó el sistema de relaciones entre variables. Se comprobaron todos los modelos con los coeficientes estandarizados obtenidos por el método de estimación de máxima verosimilitud. Puesto que el test Chi-cuadrado es sensible al tamaño de la muestra, es muy recomendable el uso de indicadores de ajuste relativo, tales como el CFI, el NNFI y el RMSA, (Bentler, 1990). Los 
valores del RMSEA menores de .05 reflejan un buen, ajuste, mientras que los valores mayores a .08 indican un ajuste pobre (Browne \& Cudeck, 1993). Los valores NNFI y CFI, mayores a .90 indican un buen ajuste (Hoyle, 1995).

Se analizaron las conexiones hipotetizadas previstas entre las necesidades básicas de los estudiantes, los enfoques de aprendizaje y el rendimiento académico (Hipótesis 1). Los valores de los índices de ajuste obtenidos $\left(N=150, \chi^{2}=42.64 ; p=.005, D . F=22 ; N F I=87\right.$; $N N F I=.88 ; C F I=.93 ; G F I=.94 ; A G F I=.88 ; R M S E A=.079)$ mostraron que el modelo se ajusta mal a los datos. Por lo que respecta a las relaciones entre variables, como se ve en Figura 2, la variable latente enfoque profundo de aprendizaje y la variable latente enfoque superficial de aprendizaje fueron bien explicadas por las necesidades básicas del estudiante $\left(R^{2}=.42\right.$ y $R^{2}=.50$, respectivamente). Sin embargo, los efectos de los enfoques de aprendizaje sobre el rendimiento de los estudiantes no fueron significativos (véase la Figure 2 para más detalles). Los datos obtenidos del modelo global indican que la primera hipótesis formulada no ha probado en su totalidad, aunque si se han observado en el modelo ciertas tendencias que transcurren en la dirección hipotetizada.

Necesidades Estudiantes (input) Enfoques de aprendizaje (proceso) Rendimiento (output)

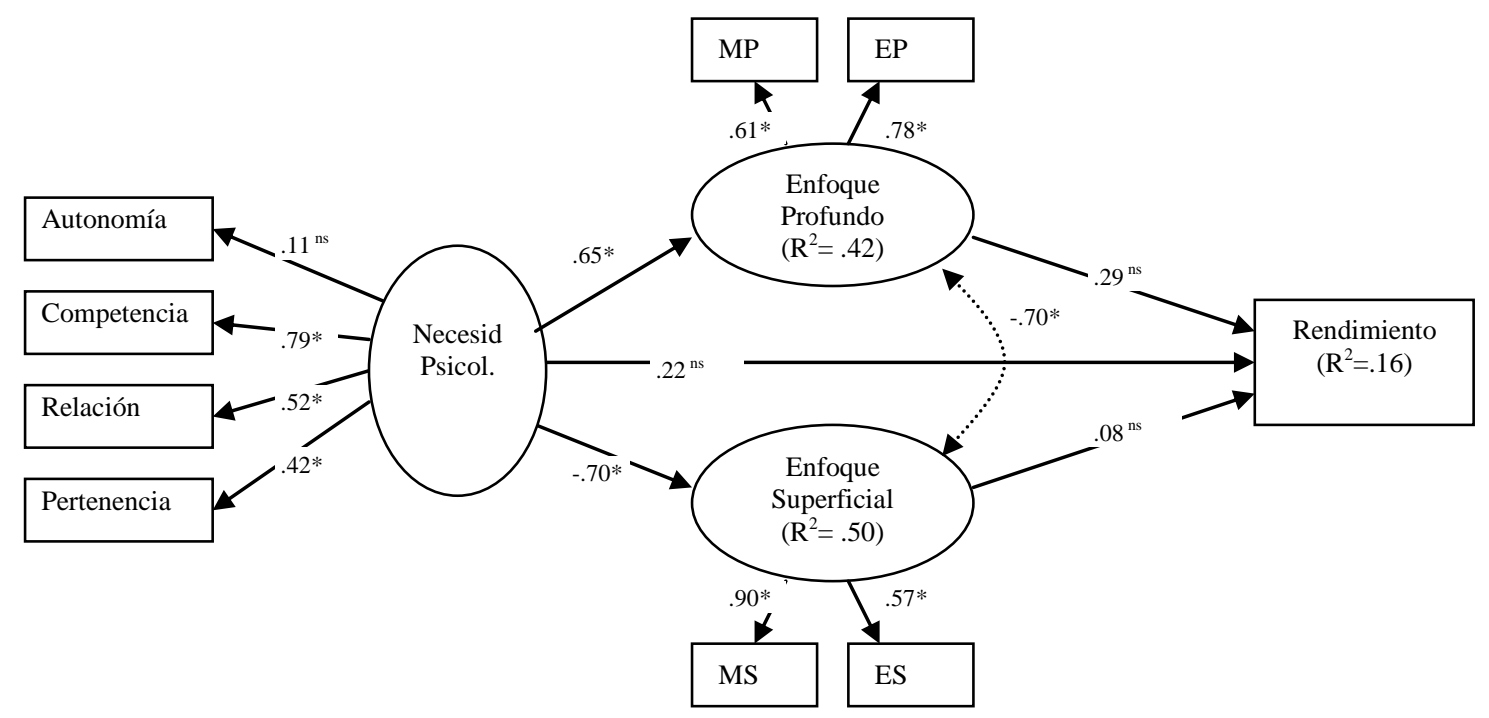

Figura 2. Relación entre necesidades psicológicas, enfoques de aprendizaje y rendimiento escolar. Se muestra la configuración estructural, coeficientes estandarizados e índices de ajuste (se han omitido algunos casos porque faltaba una variable $N=150$ casos válidos)

Nota. $*$ significativo $(p<.05), \quad \mathrm{ns}=$ no significativo

Índices de ajuste: Bondad de ajuste estadística : Chi-Cuadrado $=42.64 ;$ D.F. $=22 ; p=.005$

Bentler-Bonett Índice de ajuste normalizado $(\mathrm{NFI})=.87$ Bentler-Bonett Índice de Ajuste No Normalizado $(\mathrm{NNFI})=.88$; Indice de Ajuste Comparativo $(\mathrm{CFI})=.93$; Índice de Ajuste GFI de Lisrel = .94; Índice de Ajuste AGFI de Lisrel = .88; Raíz de la media cuadrática residual $(\mathrm{RMR})=.029$; Error cuadrático medio de aproximación $(\mathrm{RMSEA})=.079$ 
Con respecto a las segundas conexiones previstas, se examinó la relación entre la satisfacción de las necesidades básicas del alumno, las estrategias de evitación y el rendimiento (Hipótesis 2). Los valores de los índices de ajuste obtenidos $\left(N=157, \chi^{2}=30.02\right.$; $p=.03, D . F .=18 ; N F I=.86 ; N N F I=.90 ; C F I=.94 ; G F I=.95 ; A G F I=.91 ; R M S E A=.066)$ mostraron que el modelo examinado muestra un ajuste satisfactorio a los datos. Con respecto a las relaciones entre variables, la latente variable F1 (Estrategias de evitación) se tuvo en cuenta también por las necesidades básicas del estudiante $\left(R^{2}=.45\right)$. El efecto de $\mathrm{F} 1$ (Estrategias de Evitación) sobre el rendimiento académico es negativo y significativo $\left(R^{2}=\right.$ .13). Sin embargo, el rendimiento no se ve afectado por las necesidades psicológicas como se esperaba. Véase la Figura 3 para más detalles De acuerdo con los datos, podemos considerar probada la Hipótesis 2.

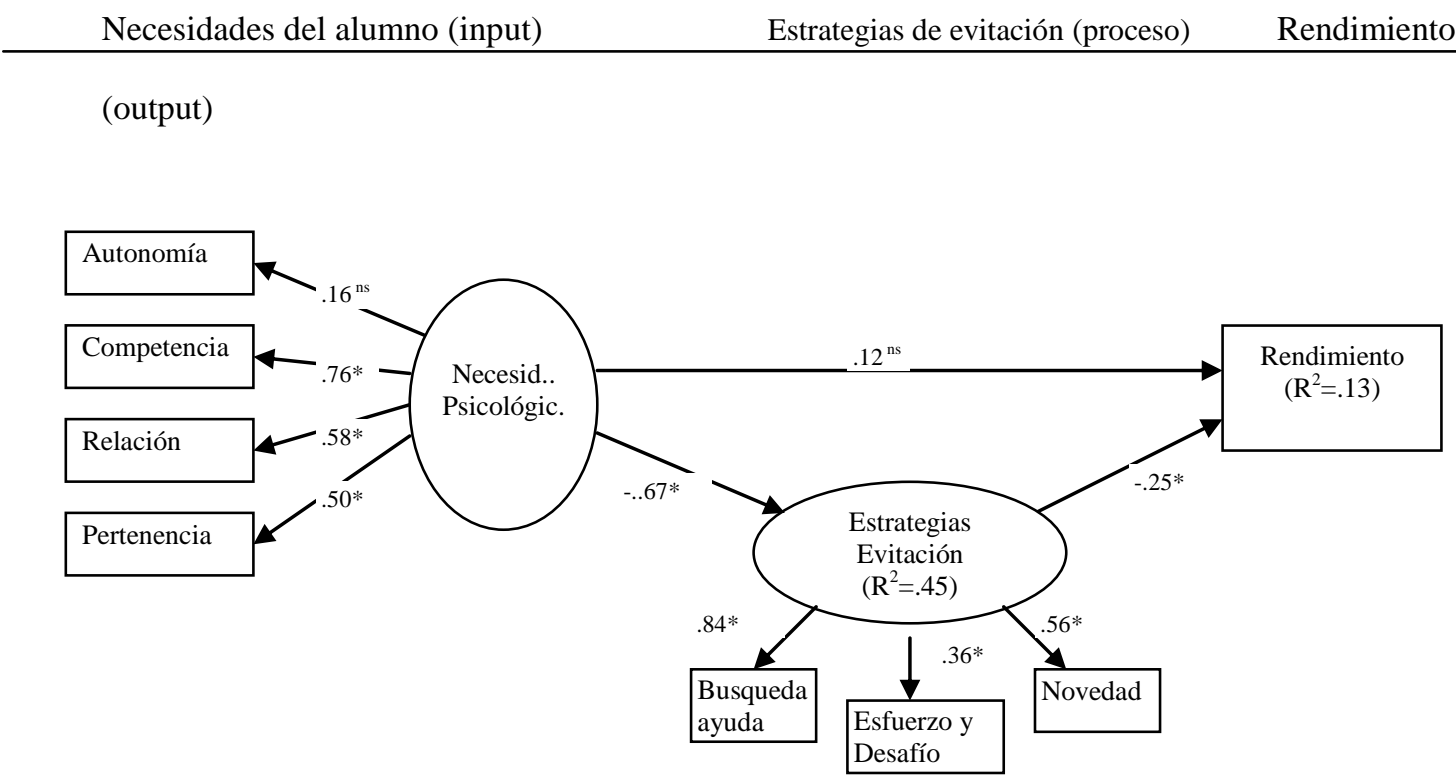

Figura 3. Relación entre necesidades, estrategias de evitación, y rendimiento. Se muestra la configuración estructural, coeficientes estandarizados e índices de ajuste. (Se han omitido algunos casos porque faltaba una variable $\mathrm{N}=157$ casos válidos) 
En relación con el modelo extendido, todas las variables se examinaron simultáneamente en un primer paso, tomando las estrategias de evitación como predictores de los enfoques de aprendizaje (Hipótesis 3), y en un segundo paso tomando los enfoques de aprendizaje como predictores de las estrategias de evitación (Hipótesis 4). Los valores de los índices fijos obtenidos para las conexiones previstas en la Hipótesis $3\left(N=139, \chi^{2}=89.57 ; p\right.$ $=.00, D . F .=47 ; N F I=.83 ; N N F I=.88 ; C F I=.91 ; G F I=.92 ; A G F I=.86 ; R M S E A=.078) \mathrm{y}$ Hipótesis $4\left(N=139, \chi^{2}=98.31 ; p=.00, D . F=47 ; N F I=.82 ; N N F I=.86 ; \mathrm{CFI}=.89 ; G F I=\right.$ $.90 ; A G F I=.84 ; R M S E A=.083)$ indicaron que el modelo se ajustaba mal a los datos. Posteriormente, y siguiendo las recomendaciones del método multiplicador de Wald y Lagrange para ajustar parámetros, se obtuvo un modelo optimizado. Los valores de los indices de ajuste $\left(N=139, \chi^{2}=62.30 ; p=.01, D . F .=39 ; N F I=88 ; N N F I=.92 ; C F I=.95 ; G F I=.94\right.$; $A G F I=.87 ; R M S E A=.063$ ) indicaron un buen ajuste a los datos. La configuración estructural, coeficientes estandarizados e índices de ajuste se muestran en la Figura 4. Los resultados revelan conexiones interesantes entre las variables que apoyan, hasta cierto punto, la relación hipotética esperada en la Figura 1. Necesidades básicas del alumno (factor input) tiene un efecto positivo significativo sobre el enfoque profundo de aprendizaje $\left(R^{2}=.31\right)$, mientras que el efecto sobre el enfoque superficial de aprendizaje es significativo pero negativo $\left(R^{2}=.32\right)$. A su vez, el enfoque superficial de aprendizaje tiene un impacto significativo y positivo sobre las estrategias de evitación $\left(\mathrm{R}^{2}=.54\right)$. Finalmente, el rendimiento recibe un impacto significativo y negativo por parte de las estrategias de evitación, y, un impacto significativo y positivo por parte del enfoque profundo de aprendizaje $\left(\mathrm{R}^{2}=.16\right)$. Estos resultados proporcionan apoyo parcial a las Hipótesis 3 y 4. 


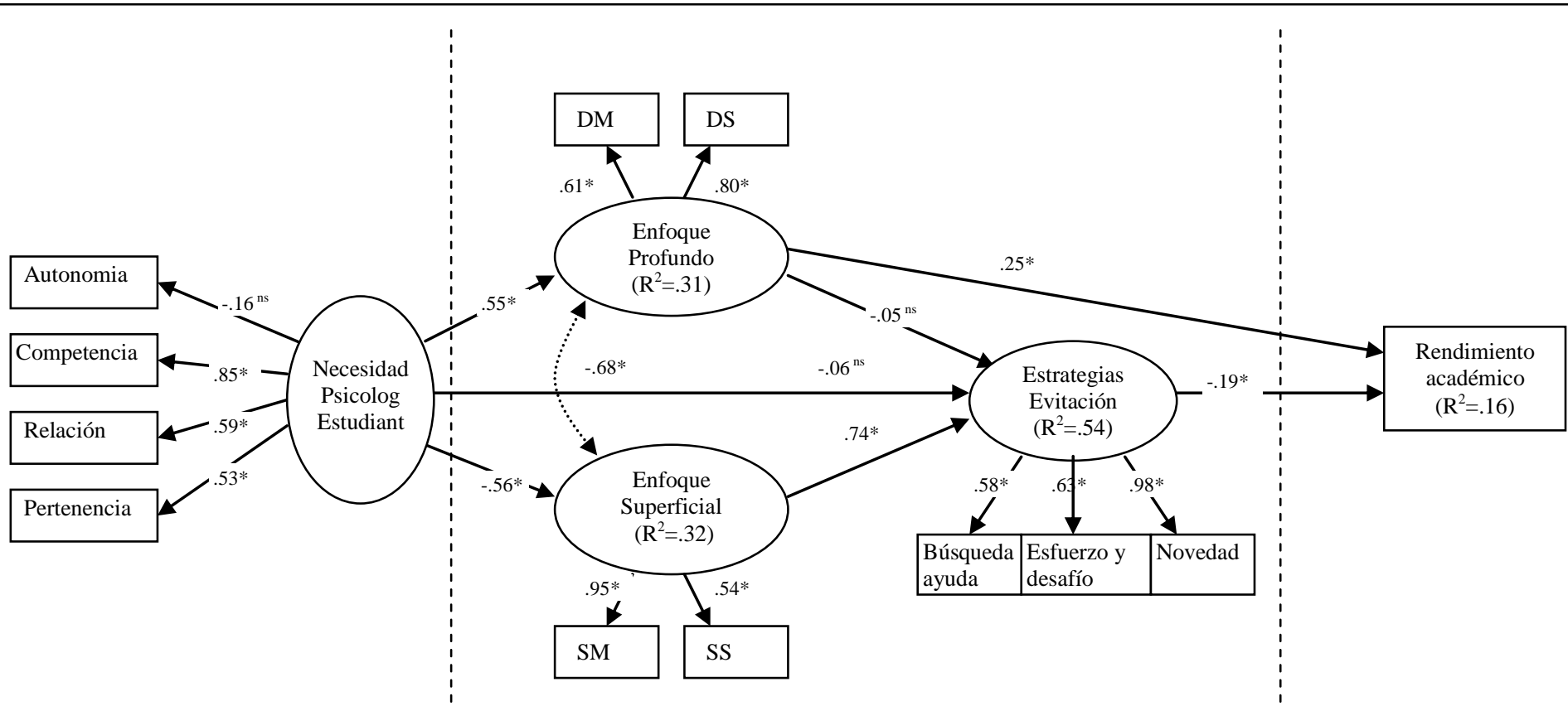

Figura 4. Modelo optimizado con respecto a la relación entre necesidades, estrategias de evitación, enfoques de aprendizaje y rendimiento académico. Se muestra la configuración estructural, coeficientes estandarizados e índices de ajuste (se han omitido algunos casos porque faltaba una variable, $\mathbf{N}=139$ casos válidos)

Nota. $*=$ significativo $(p<.05), \quad$ ns $=$ no significativo

Nota. Se estableció la covarianza entre los errores de las variables de evitación y entre los errores de las necesidades psicológicas.

Índices de ajuste: Bondad de ajuste estadística: Chi-Cuadrado $=62.30 ;$ D.F. $=39 ; p=.010$

Bentler-Bonett Índice de ajuste normalizado $(\mathrm{NFI})=.88$ Bentler-Bonett Índice de Ajuste No Normalizado $(\mathrm{NNFI})=.92$; Índice de ajuste comparativa $(\mathrm{CFI})=.95$; Índice de Ajuste GFI de Lisrel $\mathrm{x}=.94$; Índice de Ajuste AGFI de Lisrel $=.87$; Raíz de la media cuadrática residual $(\mathrm{RMR})=.031$; Error cuadrático medio de aproximación $(\mathrm{RMSEA})=.063$ 


\section{Discusión y Conclusiones}

Se argumentan las relaciones entre las necesidades psicológicas básicas del estudiante, los enfoques de aprendizaje del estudiante en el aula, su uso de las estrategias de evitación y el rendimiento en una muestra de estudiantes universitarios.

Con respecto a la primera hipótesis, el análisis estructural llevado a cabo para comprobar el modelo planteado como hipótesis reveló que, en general, las necesidades básicas del estudiante consideradas en este estudio fueron buenos predictores para los dos enfoques de aprendizaje (profundo y superficial). Sin embargo, la contribución de la autonomía es menos clara, ya que no proporciona una contribución significativa al factor de orden superior definido por las cuatro necesidades del alumno consideradas. Los coeficientes estandarizados mostraron asociaciones importantes y positivas (a excepción de la autonomía) entre las necesidades básicas y el enfoque profundo, y asociaciones negativas (a excepción de la autonomía) entre las necesidades básicas y el enfoque superficial. En general, los datos del primer modelo indican que los estudiantes que perciben sus necesidades básicas más satisfechas (a excepción de la autonomía) tienden a adoptar un enfoque profundo, y a la inversa, los estudiantes que perciben sus necesidades básicas menos satisfechas (a excepción de la autonomía) tienden a adoptar un enfoque superficial. Esta situación enfatiza la importancia de algunos aspectos instruccionales que están bajo control del profesor (método de enseñanza, tareas planteadas, diseño de la clase, interacción con los estudiantes, etcétera), ya que la decisión que tome el profesor sobre estos aspectos, mostrará a sus alumnos cuanta atención va a prestar a satisfacer sus necesidades básicas. Esto, evidentemente, repercutirá en el aprendizaje y rendimiento del alumno. Según la teoría de la autodeterminación, si las necesidades básicas están satisfechas, se estimula el bienestar psicológico y ésto permite que los estudiantes alcancen un rendimiento académico óptimo. Por el contrario, cuando estas necesidades no están satisfechas, los estudiantes no rinden académicamente (Reis, Sheldon, Gable, Roscoe, \& Ryan, 2000; Sheldon, Ryan, \& Reis, 1996; Sheldon, Elliot, Kim, \& Kasser, 2001).

Respecto a la segunda hipótesis, el análisis estructural llevado a cabo para comprobar el modelo planteado como hipótesis reveló que el modelo se ajusta bien a los datos. Como se puede ver en la Figura 3, la satisfacción de las necesidades del estudiante no tiene un impacto directo en su rendimiento, sino más bien indirecto, vía estrategias de evitación. Es decir, la 
satisfacción de las necesidades básicas tiene un efecto significativo y negativo sobre las estrategias de evitación. A su vez, las estrategias de evitación tienen un efecto significativo y negativo sobre el rendimiento. Parece que las estrategias de evitación desempeñan un rol mediador entre las necesidades de los estudiantes y su rendimiento. (Baron y Kenny, 1986). De nuevo, la necesidad de autonomía sigue sin aportar una contribución importante al factor de orden superior definido por las cuatro necesidades psicológicas como variable latente. Estudios anteriores basados en la teoría de la autodeterminación aportaron importantes pruebas sobre el impacto directo que tienen las necesidades psicológicas sobre el bienestar y la motivación, pero el mecanismo por el cual la satisfacción de las necesidades estimula el rendimiento no está tan claro. Estos resultados pueden proporcionar información valiosa para comprender el proceso subyacente que explica la relación necesidades-rendimiento.

Respecto a la tercera y cuarta hipótesis, el modelo optimizado resultante (Figura 4) reveló que la variable latente satisfacción de las necesidades del alumno se asocia a los enfoques de aprendizaje, siguiendo el mismo patrón de relación que se identificó en el primer modelo hipotetizado. A su vez, un enfoque profundo de aprendizaje se asocia de forma positiva al rendimiento vía estrategias de evitación, mientras que el enfoque superficial se asocia negativamente al rendimiento vía estrategias de evitación. Una vez más, la necesidad de autonomía no contribuye a la variable latente definida por las necesidades psicológicas. Este modelo ampliado proporciona información complementaria al modelo anterior examinado, acerca del mecanismo del proceso que conecta las necesidades psicológicas con el rendimiento, Aunque se necesita investigar más, los resultados parecen indicar que el proceso que conecta las necesidades psicológicas con el rendimiento puede explicarse, en parte, por los enfoques de aprendizaje y las estrategias de evitación. Dicho de otro modo, los enfoques de aprendizaje y las estrategias de evitación pueden desempeñar un papel mediador en la relación necesidades psicológicas-rendimiento. Es también nuestro deseo destacar el efecto negativo que el enfoque superficial tiene sobre las estrategias de evitación. Estos hallazgos sugieren que los estudiantes que adoptan un enfoque superficial no solo tienden a utilizar ciertas estrategias dirigidas a aprender de forma mecánica y repetitiva, sino que también tienden a desarrollar conductas para evitar tareas académicas nuevas, para evitar la búsqueda de ayuda y también para evitar el esfuerzo y desafío, lo que a su vez les lleva a obtener un bajo rendimiento académico. 
En contra de lo que se esperaba, parece que la necesidad de autonomía no va en la misma dirección que las demás necesidades. Los estudiantes con puntuaciones más altas en la escala de autonomía son aquellos que perciben que su profesor/a estimula el aprendizaje independiente y autónomo, mientras que los estudiantes con puntuaciones bajas representan a los que perciben que su profesor/a estimula el aprendizaje tradicional, y que su necesidad de autonomía no estaba satisfecha. Hemos encontrado tres explicaciones posibles para este hecho. La primera es que la necesidad de autonomía es ambigua, ya que el grado de autonomía que los estudiantes requieren puede cambiar en función de la etapa educativa en que se encuentren: (Primaria, Secundaria o Superior). En relación con este tema, surge una pregunta importante que justifica la necesidad de crear una escala válida: ¿Cuáles son las necesidades de los estudiantes en lo que a autonomía se refiere? o, más concretamente, ¿Qué grado de autonomía necesita un estudiante universitario?. Algunos ítems de la escala, como por ejemplo,: "Soy capaz de decidir libremente mi ritmo de aprendizaje en esta material", "el profesor ha permitido a los alumnos trabajar de manera independiente y autónoma", podrían interpretarse como que el profesor asigna tareas y luego se desentiende del aprendizaje de los estudiantes, por lo que se debería enfatizar la idea de que, aunque el profesor en realidad esté animando a los estudiantes a trabajar independientemente, él les guiará y orientará en su aprendizaje. Por tanto, cuando se redacten los ítems, en una futura revisión, sería más apropiado especificar claramente que se refieren a tareas de trabajo que implican cierta independencia pero guiadas por el profesor. La segunda explicación es que, al contrario que las otras necesidades (competencia, relación y pertenencia) que siempre se perciben como positivas, los estudiantes pueden considerar a la autonomía tanto como un elemento positivo del proceso de aprendizaje o como uno negativo, según sus variables personales tales como estilos de aprendizaje o de pensamiento. Basándose en su teoría del "autogobierno mental”, Sternberg (1997) introdujo el concepto de estilos de pensamiento. La teoría de los estilos de pensamiento se refiere a qué es lo que una persona quiere hacer y cómo lo quiere hacer. Por ejemplo, según Sternberg (1997), a las personas ejecutivas les gusta que les digan qué tienen que hacer o como deben hacerlo. Prefieren trabajar en tareas estructuradas y con instrucciones claras. Por lo contrario, a las personas legislativas les gusta hacer las cosas a su manera y prefieren trabajar en proyectos que requieran estrategias creativas. Respecto a este tema, surgen dos cuestiones importantes, ¿Qué estilo de pensamiento predomina entre los estudiantes universitarios españoles? (ciertos estudiantes universitarios españoles podrían preferir una metodología de enseñanza tradicional, relacionada con el estilo ejecutivo, según García-Ros, Pérez-González, y Talaya, 2008), ¿Y cómo se relacionan con los enfoques de 
aprendizaje? Según la teoría de Sternberg (1997), las personas se sienten más cómodas y son mas eficaces cuando su estilo de pensamiento predominante se ajusta bien a las exigencias del contexto y en este caso, con la forma en que se organiza y desarrolla el curso o, dicho de otro modo, con el estilo/s de enseñanza empleados por el profesor (Doménech-Betoret, 2007). Por lo tanto, desde esta perspectiva, la autonomía podría actuar como un estímulo que activara el interés intrínseco y la implicación con la materia de estudio, o todo lo contrario. La tercera se refiere a la teoría de la evaluación cognitiva (TEC). La TEC fue presentada por Deci y Ryan (1985) como una subteoría dentro de la teoría de la autodeterminación. La TEC especifica que el sentimiento de competencia no estimula la motivación intrínseca (relacionada con el enfoque profundo) a menos que vaya acompañada de un sentido de autonomía (Ryan \& Deci, 2000). También es posible que el sentido de autonomía no estimulara la motivación intrínseca (relacionada con el enfoque profundo) a menos que estuviese acompañada de un sentido de competencia. Covington (1992) afirmó que la búsqueda de la autoaceptación es la mayor prioridad del ser humano y que "en los colegios, la autoaceptación pasa a depender de la capacidad que uno tiene para conseguir buenos resultados académicos” (p.74). Estas últimas consideraciones pueden sugerir que quizás el sentimiento de autonomía no sea suficiente para hacer que los estudiantes adopten un enfoque profundo del aprendizaje, cuando se trate de motivación intrínseca, sino que más bien podría ser el resultado de la interacción entre la autonomía y otras variables individuales tales como competencia (o auto eficacia) o estilo de pensamiento

En síntesis, este estudio destaca la importancia de apoyar las necesidades de los estudiantes en el aula, ya que están unidas a la motivación intrínseca (Deci, 1971). En general, se ha constatado que cuando se satisfacen las necesidades básicas de los estudiantes, se promueve la utilización de enfoques de aprendizaje profundo, lo que a su vez repercute en una menor utilización de estrategias de evitación. Por el contrario, cuando los estudiantes no perciben sus necesidades psicológicas satisfechas se está promoviendo la utilización de enfoques de aprendizaje superficial, que a su vez repercute en una mayor utilización de estrategias de evitación y, como consecuencia, en un menor rendimiento escolar.

El entorno de aprendizaje de la educación superior se caracteriza por un curriculum sobrecargado, y la presión por superarlo con éxito; situación que puede incluso impedir que los estudiantes se interesen por la asignatura. Esta situación puede llevar a muchos estudiantes a adoptar estrategias de aprendizaje a corto plazo (Newble \& Hejka, 1991) que están más de 
acuerdo con el enfoque estratégico (Entwistle \& Waterson, 1988) y con el enfoque superficial. Basándonos en las pruebas empíricas proporcionadas por este estudio, esta desventaja se podría reducir o mitigar, con el apoyo del profesor a las necesidades básicas del alumno, especialmente a la competencia, ya que es la variable que tiene una mayor contribución al factor de orden superior al que pertenece.

Los resultados obtenidos tienen también implicaciones prácticas en la enseñanza. Si los profesores quieren que sus alumnos se involucren en sus tareas de aprendizaje de manera que aumente el significado y la comprensión, entonces sería beneficioso crear entornos de aprendizaje que apoyen y trabajen las necesidades psicológicas básicas. Dicho de otro modo, los profesores pueden facilitar el aprendizaje comprensivo creando entornos de aprendizaje de apoyo, estableciendo con los estudiantes una relación amable y cordial, tratándoles con respeto, ofreciéndoles la oportunidad de que tomen mayor responsabilidad en su propio aprendizaje, y creando un "sentimiento de grupo" dentro del aula. La provisión de un entorno de aprendizaje en el aula que refleje estas condiciones no solo facilitaría la adopción de enfoques profundos de aprendizaje, sino que además reducirá el uso de estrategias de evitación.

\section{Limitaciones}

Deberían tomarse en cuenta varias limitaciones. La primera de ellas es la que se refiere al uso de autoinforme en todos los constructos. Los expertos recomiendan que este tipo de medida se combine con otras medidas más cualitativas u observacionales. La segunda limitación importante está relacionada con la generalización de estos hallazgos. Este trabajo de investigación se llevó a cabo en un marco educativo concreto con unas asignaturas de educación superior concretas. Por lo tanto, son necesarios más estudios de replicación en otras asignaturas y en otras etapas educativas para encontrar regularidades en contextos educativos similares (misma área de conocimiento y misma etapa educativa). Por tanto, debemos ser cautelosos en la generalización de estos resultados. En tercer lugar, se requiere una secuencia temporal de medición a fin de establecer una relación rigurosa de causa-efecto, para ello son necesarios estudios longitudinales. Finalmente señalar que, además de los enfoques profundo y superficial, sería interesante incluir también el enfoque estratégico (Entwistle \& Waterson, 1988), ya que es el mayor predictor de rendimiento académico en estudios superiores (Diseth 
\& Martinsen, 2003; Newble \& Hejka, 1991), y tal vez también esté más estrechamente relacionado con las estrategias de evitación

\section{Futuras investigaciones}

A pesar de sus limitaciones, este trabajo amplia los resultados de trabajos anteriores en las relaciones entre necesidades básicas de los estudiantes, enfoques de aprendizaje, estrategias de evitación y rendimiento, aunque se necesite investigación adicional en la que se utilice tanto una metodología cuantitativa como una cualitativa. Sería beneficioso para futuras investigaciones introducir necesidades adicionales de los estudiantes que no se hayan considerado en este trabajo, por ejemplo la necesidad de cognición (Cacioppo \& Petty, 1982) y la necesidad de control sobre el aprendizaje (Eshel \& Kohavi, 2003). Por otra parte, sería aconsejable revisar y reformular los ítems de la escala que mide la necesidad de autonomía, donde se especifique con claridad que nos referimos a un aprendizaje de carácter autónomo pero a la vez guiado por el profesor. También sería interesante que en el futuro otras investigaciones tuviesen en cuenta otros constructos mediadores relacionados con la motivación del estudiante como las metas de ejecución o rendimiento (Roeser, Midley, \& Urban, 1996), o también desorganización, perseverancia y esfuerzo, las cuales pueden afectar el rendimiento académico (Phan, 2009). Los modelos deberían completarse añadiendo resultados de tipo procedimental (no solo declarativos) que configurarían nuevas variables criterio. Finalmente subrayar que la mayoría de la investigación sobre necesidades realizada en el campo de la educación se ha llevado a cabo en escenarios de laboratorio, mientras que la investigación experimental en el aula desarrollada en asignaturas concretas es escasa. Se necesita investigar más para saber si el papel que juega la autonomía es similar en contextos diferentes y también en diferentes etapas de la vida. 


\section{References}

Baron, R. M. \& Kenny, D. A. (1986). The moderator-mediator variable distinction in social psychology research: Conceptual, strategic, and statistical considerations. Journal of Personality and Social Psychology, 51, 1173-1182.

Baumeister, R. F., \& Leary, M.R. (1995). The need to belong: Desire for interpersonal attachments as a fundamental human motivation. Psychological Bulletin, 117, 497-529.

Bentler, P. M. (1995). EQS Structural equations program manual. Encino, CA: Multivariate Software, Inc.

Bentler, P. M. (1990). Comparative fix indexes in structural models. Psychological Bulletin, 107, 238-246.

Biggs, J. (1987). Students approaches to learning and studying. Melbourne, Australia: Australian Council for Educational Research.

Biggs, J. (1989). Approaches to the enhancement of tertiary teaching. Higher Education Research and Development. 8, 7-25.

Biggs, J., \& Moore, P. (1993). The process of learning ( $3^{\text {rd }}$ ed.). New York: Prentice Hall.

Biggs, J., Kember, D., \& Leung, D.Y.P. (2001). The revised two-factor study Process questionnaire: R-SPQ-2F. British Journal of Educational Psychology, 71, 133-149.

Browne, M. W., \& Cudeck, R. (1993). Alternative ways of assessing model fit. In K. A. Bollen \& J. S. Long (Eds.), Testing Structural Equation Models. Beverly Hills, CA: Sage, 132-162.

Cacioppo, J. T., \& Petty, R. E. (1982). The need for cognition. Journal of Personality and Social Psychology, 42,116-131.

Cleave-Hogg, D., \& Rothman, A. I. (1991). Discerning views: medical students' perceptions of their learning environment. Evaluation and the Health Professions, 14, 456-474.

Covington, M. V. (1992). Making the grade: A self-worth perspective on motivation and school reform. New York: Cambridge University Press.

Dart, B. C., Burnett, P. C., Boulton-Lewis, G., Campbell, J., Smith, D., and McCrindle, A. (1999). Classroom learning environment and students' approaches to learning. Learning Environments Research, 2, 137-156.

Dart, B. C., Burnett, P. C., Purdie, N., Boulton-Lewis. G., Campbell, J.\& Smith, D. (2000). Students' conceptions of learning, the classroom environment, and approaches to learning. Journal of Educational Research, 93, 4, 262-272. 
Deci, E. (1971). The effect of externally mediate rewards on intrinsic motivation. Journal of Personality and Social Psychology, 18, 105-115.

Deci, E., \& Ryan, R. (1985). Intrinsic motivation and self-determination in human behavior. New York: Plenum.

Deci, E., \& Ryan, R. (2000). The "what" and "why" of goal pursuits: Human needs and the self-determination of behavior. Psychological Inquiry, 11, 227-268.

Diseth, A., Martinsen, O. (2003). Approaches to learning, Cognitive Style, and Motives as Predictors of Academic Achievement. Educational Psychology, 23, 196-207.

Doménech-Betoret, F. (2007). The influence of students' and teachers' thinking styles on student course satisfaction and on their learning process. Educational Psychology, 27, 219-234.

Doyle, W. (1977). Paradigms for research on teacher effectiveness. In L. Shulman (Ed.), Review of Research inEducation (Vol. 5; pp. 163-198). Ithaca, IL: Peacock.

Entwistle, N. J. (1987). Understanding classroom learning. London: Hodder and Stoughton.

Entwistle, N. J., \& Waterson, S. (1988). Approaches to studying and levels of processing in university students. British Journal of Educational Psychology, 58, 258-265.

Eshel, Y., \& Kohavi, R. (2003). Perceived classroom control, self-regulated learning strategies, and academic achievement. Educational Psychology, 23, 249-260.

Filak, V. F., \& Sheldon, K. M. (2003). Student psychological need satisfaction and college teacher-course evaluation. Educational Psychology, 23(3), 235-247.

Fraser, B. (1989). Twenty years of classroom climate work: progress and prospect. Journal of Curriculum studies, 21, 307-327.

Fraser, B. (1998). Science learning environments: assessment, effects and determinants. In B.

J. Fraser \& K. G. Tobin (Eds.), International handbook of science education (pp. 527564). Dordrecht, The Netherlands: Kluwer.

Gagné, M., \& Deci, E. L. (2005). Self-determination theory and work motivation. Journal of Organizational Behavior, 26, 331-362.

García-Ros, R., Pérez-González, F., Talaya, I. (2008). Preferencias respecto a métodos instruccionales de los estudiantes universitarios de nuevo acceso y su relación con estilos de aprendizaje y estrategias motivacionales. Electronic Journal of Research in Educational Psychology, 6, (3), 547-570.

Goodenow, C. (1992). Strengthening the links between educational psychology and the study of social context. Educational Psichologist, 27, 177-196. 
Hoyle, R. H. (1995). The structural equation modeling approach: Basic concepts and fundamental issues. In R. H. Hoyle (Ed.), Structural equation modeling, concepts, issues, and applications (pp. 1-15). Thousand Oaks, CA: Sage.

Ilardi, B. C., Leone, D., Kasser, R., \& Ryan, R.M. (1993). Employee and supervisor rating of motivation: Main effects and discrepancies associated with job satisfaction and adjustment in a factory setting. Journal of Applied Social Psychology, 23, 1789-1805.

Justicia, F., Pichardo, M. C., Cano, F., Berbén, A. B. G., De la Fuente, J. (2008). The Revised Two-Factor Study Process Questionnaire (R-SPQ-2F): Exploratory and confirmatory factor analyses at item level. European Journal of Psychology of Education, 23 (3), 355-372.

Leung, D. Y. P., \& Kember, D. (2003). The relationship between approaches to learning and reflexion upon practice. Educational Psychology, 23, 61-71.

Lord, F.M., \& Novick, M. R. (1968). Statistical theories of mental test scores. Reading: Addison-Wesley.

Newble, D., \& Hejka, E. J. (1991). Approaches to learning of medical students and practising physicians: Some empirical evidence and its implication for medical education. Educational Psychology, 11, 333-342.

Nichols, J. C., \& Miller, A. T. (1984). Developments and its discontents: The differences of the concept of ability. In J. G. Nicholls (Ed.), Advances in motivation and achievement (pp. 185-218). Greenwich, CT: JAI Press.

Norusis, M.J. (1999). SPSS for Windows (Versión 10.0). Chicago; IL: SPSS Inc.

Phan, H. P. (2009). Pensamiento reflexivo, persistencia, desorganización y ejecución académica: un enfoque mediacional. Electronic Journal of Research in Educational Phychology, 7(3), 927-952.

Ramsden, P. (1992). Learning to teach in higher education. London: Routledge.

Ramsden, P., Martin, E., \& Bowden, J. ( 1989). School environment and sixth form pupils' approaches to learn. British Journal of Educational Psychology, 59, 129-142.

Ramsden, P., \& Entwistle, N. (1981). Effects of academic departments on students' approaches to studying. British Journal of Educational Psychology, 51, 367-383.

Reis, H., Sheldon, K., Gable, S., Roscoe, J., \& Ryan, R. (2000). Daily well-being: The role of autonomy, competence and relatedness. Personality and Social Psychology Bulletin, 26, 419-435. 
Roeser, R. W., Midgley, C., \& Urdan, T. C. (1996). Perceptions of school psychological environmental and early adolescents' psychological and behavioral functioning in school: the mediating role of goals and belonging. Journal of Educational Psychology, $88,3,408-422$.

Ryan, M. R., Connell, J. P., \& Grolnick, W. S. (1992). When achievement is not intrinsically motivated: A theory of internalization and self-regulation in school. In A. K. Boggiano \& T. S. Pittman (Eds.), Achievement and motivation. A social-developmental perspective (pp. 167-188). New York: Cambridge University Press.

Ryan, M. R., \& Deci, E. (2000). Self-determination theory and the facilitation of intrinsic motivation, social development, and well-being. American Psychologist, 55, 68-78.

Ryan, R., \& Stiller, J. (1991). The social contexts of internalization: Parents and teacher influences on autonomy, motivation and learning. In P.R. Pibtrich \& M. L. Maehr (Rds), Advances in motivation and achievement, 7. Goals and self-regulating process (pp. 115-149), Greenwich, CT: JAI Press.

Schmeck, R. R. (1988). Strategies and styles of learning: An integration of varied perspectives. In Ronald Ray Schmeck (Ed) Learning strategies and learning styles, pp. 317-347. New York, NY, US: Plemun Press.

Sheldon, K. M., Elliot, A.J., Kim, Y., \& Kasser, T. (2001). What's satisfying about satisfying events? Comparing ten candidate psychological needs. Journal of Personality and Social Psychology, 80, 325-339.

Sheldon, K., \& Biddle, B. (1998). Standards, accountability and school reform: Perils and pitfalls. Teacher College Record, 100, 164-180.

Sheldon, K., Ryan, R., \& Reis, H. (1996). What makes for a good day? Competence and autonomy in the day and in the person. Personality and Social Psychology Bulletin, 22, 66-84.

Schmitt, N. (1996). Uses and abuses of coefficient alpha. Psychological Assessment, 8, 350353.

Sternberg, R. J. (1997). Thinking Styles. Cambridge University Press.

Turner, J. C., Meyer, D. K., Anderman, E. M., Midgley, C., Gheen, M., \& Kang, Y. (2002). The classroom environment and students' reports of avoidance strategies in mathematics: a multimethod study. Journal of Educational Psychology, 94, 1, 88-106. 
Valas, H., \& Sovik, N. (1993). Variables affecting students' intrinsic motivation for school mathematics: two empirical studies based on Deci and Ryan's theory on motivation. Learning and Instruction, 3, 281-298

Waxman, H. (1991). Investigating classroom and school learning environments: a review of recent research and developments in the field. Journal of Classroom Interaction, 26(2), $1-4$.

Yuen-Yee, G. C. \&Watkins, D. (1994). Classroom environment and approaches to learning: an investigation of the actual and preferred perceptions of Hong Kong secondary school students. Instructional Science, 22, 233-246. 
Escala de las necesidades básicas del estudiante (4 escalas, 17 ítems)

Escriba delante de cada ítem el número que considere más apropiado:

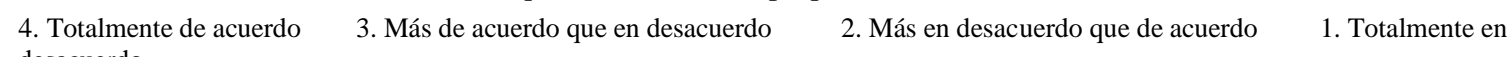
desacuerdo

a) Autonomía $(\alpha=0.76)$.

..... 1. He podido decidir libremente mi ritmo de aprendizaje.

..... 2. He podido elegir libremente las tareas que debía hacer para estudiar esta asignatura.

...... 3. El profesor ha permitido a los estudiantes trabajar de forma independiente.

...... 4. He sentido que podía decidir sobre cómo trabajar y aprender esta asignatura.

b) Competencia $(\alpha=0.65)$.

..... 1. Cuando estudiaba esta materia me sentía capaz de hacerlo

..... 2. He tenido la oportunidad de demostrar mi capacidad durante el proceso de aprendizaje de esta asignatura.

..... 3. Me he sentido suficientemente competente para afrontar el desafío y los trabajos planteados en el aprendizaje de esta asignatura.

..... 4. He podido aprender destrezas nuevas e interesantes en esta asignatura

c) Relación $(\alpha=0.82)$.

..... 1. El profesor me dio la suficiente confianza como para que yo le preguntara cualquiera cosa.

..... 2. El profesor ha sido amable y cordial conmigo.

...... 3. He tenido la sensación de que el profesor era amable y quería ayudarme

...... 4.El profesor ha sido muy comprensivo (se ponía en el lugar del otro/a) con los problemas de los estudiantes.

d) Pertenencia $(\alpha=0.86)$.

..... 1. Hay un fuerte sentimiento de amistad en este grupo

..... 2.Me he sentido a gusto en este grupo/clase

..... 3. Ser parte de este grupo es como pertenecer a una gran familia.

..... 4.Tengo la sensación de que en esta asignatura somos un gran equipo

..... 5. En el futuro recordaré a mis compañeros/as de este grupo/clase con cariño.

Enfoque de aprendizaje del estudiante (4 escalas, 14 ítems)

a) Escala de Motivación Profunda (MP) (3 ítems)

.... 5. Creo que cualquier tema de esta asignatura puede ser interesante si le prestas toda tu atención

..... 9. Me he dado cuenta de que, en general, los temas de esta asignatura pueden resultar tan interesantes como un buen libro

.... 1. Me ha producido satisfacción estudiar y trabajar los contenidos de esta asignatura

b) Escala de Estrategia Profunda (EP) (4 ítems)

.... 2. He descubierto que para sentirme satisfecho en esta asignatura, he tenido que trabajar mucho en un tema determinado para sacar mis propias conclusiones

.... 18. Para comprender bien la materia le preguntaba al profesor sobre algunos temas

.... 14. He invertido parte de mi tiempo en buscar más información sobre temas interesantes que se debían de haber visto en clase.

.... 6. He dedicado mucho tiempo a profundizar y ampliar los apuntes de clase.

c) Escala de Motivación Superficial. Escala de Motivación Superficial (MS) (4 ítem)

.... 12. En general, en esta asignatura me he limitado a hacer lo que se esperaba de mí, porque creía que no era necesario hacer más esfuerzo

.... 4. En esta asignatura no he intentado hacer más de lo que se esperaba de mí, ni ningún esfuerzo mayor del necesario.

.... 3. Mi intención en esta asignatura era sacar la mayor nota posible con el mínimo esfuerzo.

.... 17. Mi objetivo principal era aprobar la asignatura, me daba igual aprender más o menos. 


\section{d) Escala de Estrategia Superficial (ES) (3 ítems)}

.... 8. Cuando estudiaba esta asignatura, intentaba memorizar los contenidos, aunque a veces no los comprendiera.

.... 11. He descubierto que la mejor manera de sacar notas altas en los exámenes de esta asignatura es memorizando.

.... 20. He intentado memorizar las respuestas a las preguntas que podían salir en el examen.

Escala de Estrategias de Evitación (3 escalas, 15 ítems)

Escriba delante de cada ítem el número que considere más apropiado:

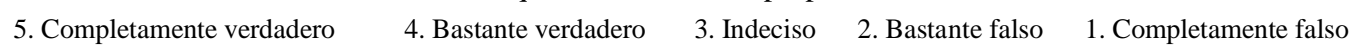

a) Evitación de las situaciones nuevas $(\alpha=0.76)$.

..... 1. De los diferentes trabajos optativos propuestas en esta materia, solía elegir aquellos que sabía que podía hacer sin mucha dificultad, y evitaba elegir los trabajos más complejos.

..... 2. En esta asignatura, prefiero hacer tareas que ya conozco antes que intentar otras nuevas que todavía he de aprender cómo hacerlas.

..... 3. En esta asignatura, prefiero trabajar aquellos contenidos que ya sé en vez de intentar con otros que todavía no sé

..... 4.No me gustaría tener que aprender contenidos nuevos en esta asignatura.

...... 5. Prefiero los trabajos que sé cómo hacer a aquellos que incluyen contenidos y procedimientos nuevos.

b) Evitación de la búsqueda de ayuda $(\alpha=0.73)$.

...... 1. Si hay algo que no entiendo de esta asignatura, intento solucionarlo solo en vez de pedir ayuda.

..... 2. En esta asignatura, no pregunto en clase aunque no entienda lo que estén explicando.

..... 3. Cuando no entiendo las tareas o actividades que el profesor propone, intento hacerlas como yo creo que están bien pero no pido ayuda.

..... 4. Aunque las tareas me resulten muy difíciles para hacerlas yo solo no suelo pedir ayuda.

...... 5. Si las tareas que propone el profesor son demasiado difíciles para mí, opto por no hacerlas o por copiarlas, pero no pido ayuda.

c) Evitación del esfuerzo y el desafio $(\alpha=0.84)$.

...... 1. Mi prioridad ha sido superar la asignatura con el mínimo esfuerzo.

..... 2. He optado por hacer tareas que eran más fáciles para mí, y he evitado aquellas que suponían un desafío.

..... 3. He preferido hacer las actividades y los trabajos de esta asignatura que me suponían el menor esfuerzo.

..... 4. En esta asignatura no he querido afrontar desafíos importantes y trabajos difíciles.

...... 5. En esta asignatura he hecho solo lo que se esperaba de mí. 